\title{
Short Communication UGT1A1*28 Variant Allele Is a Predictor of Severe Hyperbilirubinemia in HIV-Infected Patients on HAART in Southern Brazil
}

\author{
Lisiane Turatti, Eduardo Sprinz, ${ }^{2}$ Rosmeri K. Lazzaretti, ${ }^{2}$ Regina Kuhmmer, ${ }^{2}$ Grasiela Agnes, \\ Jussara M. Silveira, ${ }^{3}$ Rossana P. Basso, ${ }^{3}$ Cezar A.T. Pinheiro, ${ }^{4}$ Mariângela F. Silveira, ${ }^{4}$ \\ Silvana de Almeida, Jorge P. Ribeiro, ${ }^{2}$ and Vanessa S. Mattevi ${ }^{1}$
}

\begin{abstract}
Highly active antiretroviral therapy (HAART) has increased the survival of HIV-infected patients. However, adverse effects play a major role in adherence to HAART. Some protease inhibitors (mainly atazanavir and indinavir) act as inhibitors of uridine diphosphate-glucuronosyltransferase (UGT1A1), the enzyme responsible for hepatic conjugation of bilirubin. Variations in the promoter region of the UGT1A1 gene (UGT1A1*28, rs8175347) can influence bilirubin plasma levels, modulating the susceptibility to hyperbilirubinemia. Aiming to analyze the association between UGT1A1*28 allele and hyperbilirubinemia in individuals exposed to HAART, we evaluated $375 \mathrm{HIV}$-positive individuals on antiretroviral therapy. Individuals carrying the UGT1A1*28 allele had a higher risk of developing severe hyperbilirubinemia [prevalence ratio $(\mathrm{PR})=2.43,95 \%$ confidence interval (CI) 1.08-5.45, $p=0.032]$ as well as atazanavir users ( $P R=7.72,95 \% \mathrm{CI}=3.14-18.98, p<0.001)$. This is the first description of such an association in Brazilian HIV patients, which shows that in African-American and Euroamerican HAART users, the UGT1A1*28 allele also predisposes to severe hyperbilirubinemia, especially in those exposed to atazanavir.
\end{abstract}

$\mathbf{H}$ IGHLY ACTIVE ANTIRETROVIRAL therapy (HAART) has completely changed the prognosis of HIV-infected individuals. ${ }^{1,2}$ However, drug toxicity and adverse effects are still major challenges to treatment success. Nevertheless, not all people exposed to the same antiretrovirals may present the same adverse effect, and genetic variations between humans might be responsible for the difference rates and expression of these adverse events. ${ }^{1}$

Hyperbilirubinemia is one of these adverse effects and the development of jaundice could lead to adherence problems and treatment failure. ${ }^{3,4}$ This is particularly true in HAART regimens containing indinavir, and, mainly, atazanavir (ATV), which inhibit the enzyme uridine diphosphate-glucuronosyltransferase A family, polypeptide A1 (UGT1A1). ${ }^{4-6}$ The development, frequency, and severity of hyperbilirubinemia differ between individuals and one of the possible explanations might be related to genetics. ${ }^{4-6}$ The combination of genetic variants added to exposure to xenobiotics and environmental factors can influence the activity of glucuronidation. ${ }^{4}$
UGT1A1 gene promoters containing seven TA repeats $\mathrm{A}(\mathrm{TA})_{7} \mathrm{TAA}$, also known as UGT1A1*28 (rs8175347), cause a reduction of approximately $50 \%$ in enzyme activity, in comparison with the wild-type six TA repeats-containing allele $\left(U G T 1 A 1^{*} 1\right){ }^{7}$ Also, the UGT1A1*28 polymorphism has been related to adverse drug effects such as toxicity and predisposition to cancer. ${ }^{4}$ Other rarer variants also reduce (eight repeats, UGT1A1*37) or increase (five repeats, UGT1A1*36) enzyme activity. ${ }^{7}$ Based on this rationale, this study aimed to analyze the association between the UGT1A1*28 allele and the frequency and severity of hyperbilirubinemia in HIV-infected patients on HAART, previously unexplored in Brazilian patients.

We developed a cross-sectional study in which we analyzed consecutively 375 HIV-infected individuals in governmentsupported reference treatment services in three different cities (Porto Alegre, Pelotas, and Rio Grande) from the Brazilian southernmost state. This study included only people aged over 18 years, with viral load below the detection limit of the test

\footnotetext{
${ }^{1}$ Universidade Federal de Ciências da Saúde de Porto Alegre-UFCSPA, Porto Alegre, RS, Brazil.

${ }^{2}$ Hospital de Clínicas de Porto Alegre-HCPA, Porto Alegre, RS, Brazil.

${ }^{3}$ Hospital Universitário Dr. Miguel Riet Correa Jr., Universidade Federal do Rio Grande, Rio Grande, RS, Brazil.

${ }^{4}$ Serviço de Assistência Especializada em HIV/AIDS, Universidade Federal de Pelotas, Pelotas, RS, Brazil.
} 
Table 1. Median Bilirubin Levels Compared Between the Common UGT1A1 rs8175347 Genotypes

\begin{tabular}{llcl}
\hline Genotypes & \multicolumn{1}{c}{ Total bilirubin } & $\mathrm{p}$ & \multicolumn{1}{c}{ Indirect bilirubin } \\
\hline $\mathrm{TA}_{6} / \mathrm{TA}_{6}(n=169)$ & $0.67 \mathrm{mg} / \mathrm{dl}(\mathrm{IQR}, 0.40-1.20 \mathrm{mg} / \mathrm{dl})$ & $0.848^{\mathrm{a}}$ & $0.47 \mathrm{mg} / \mathrm{dl}(\mathrm{IQR}, 0.30-0.89 \mathrm{mg} / \mathrm{dl})$ \\
$\mathrm{TA}_{6} / \mathrm{TA}_{7}(n=145)$ & $0.61 \mathrm{mg} / \mathrm{dl}(\mathrm{IQR}, 0.40-1.23 \mathrm{mg} / \mathrm{dl})$ & & $0.40 \mathrm{mg} / \mathrm{dl}(\mathrm{IQR}, 0.30-0.87 \mathrm{mg} / \mathrm{dl})$ \\
$\mathrm{TA}_{7} / \mathrm{TA}_{7}(n=39)$ & $0.80 \mathrm{mg} / \mathrm{dl}(\mathrm{IQR}, 0.40-1.30 \mathrm{mg} / \mathrm{dl})$ & & $0.50 \mathrm{mg} / \mathrm{dl}(\mathrm{IQR}, 0.30-1.00 \mathrm{mg} / \mathrm{dl})$ \\
Whole sample $(n=353)$ & $0.67 \mathrm{mg} / \mathrm{dl}(\mathrm{IQR}, 0.40-1.20 \mathrm{mg} / \mathrm{dl})$ & & $0.40 \mathrm{mg} / \mathrm{dl}(\mathrm{IQR}, 0.30-0.85 \mathrm{mg} / \mathrm{dl})$ \\
\hline
\end{tabular}

${ }^{a}$ Kruskal-Wallis test.

$\mathrm{IQR}$, interquartile range.

(bDNA, 50 copies $/ \mathrm{ml}$ ) and using antiretroviral therapy regularly for at least 1 year. Indirect and total bilirubin levels were measured through standard methods in each service. For the definition of hyperbilirubinemia, the AIDS Clinical Trials Group guideline criterion was used. ${ }^{8}$ All patients signed the Free and Informed Consent Form. This study was approved by the Research Ethics Committees from the institutions involved.

Genomic DNA was extracted from leukocytes by a standard salting out methodology. The fragment containing the chromosomal region of interest of the UGT1A1 gene was amplified by polymerase chain reaction (PCR), using the primers described by Smiderle et al. ${ }^{9}$ The size of the amplicons was determined by capillary electrophoresis on the ABI PRISM 310 Genetic Analyzer (Applied Biosystems, USA).

To check whether the genotype frequencies were in agreement with those expected under Hardy-Weinberg equilibrium (HWE), the Roff and Bentzen (1989) chi-square test was used. ${ }^{10}$ Due to their asymmetric distribution, mean bilirubin levels were compared among genotypes by KruskalWallis tests. Poisson regression models with robust variance were used to assess the predictor variables for the development of severe hyperbilirubinemia. The variables ethnic group, gender, indinavir use, atazanavir use, age, and presence of the UGT1A1*28 allele were included in the regression model and removed stepwise. Only those that were significant predictors were kept in the final model. Statistical analyses were performed with Statistical Package for Social Sciences Version 16.0 (SPSS, Chicago, IL). Differences were considered significant when $p<0.05$.

We analyzed a total of 375 HIV-infected individuals: $60.3 \%$ were Euro-Brazilians and 39.7\% Afro-Brazilians, classified according to the phenotypic definition by the interviewer. ${ }^{11}$ Males comprised $54.4 \%$ of our sample. The mean age was $43.2 \pm 9.6$ years. The median time on antiretrovirals was 58.0 months (interquartile range, IQR, 34 to 105 months). Regarding treatment, $51.7 \%$ were protease inhibitor (PI) users. ATV users comprised $25.1 \%$ (94) of our sample and IDV users comprised $1.6 \%(6)$.

UGT1A1 allele frequencies were 0.015, 0.665, 0.306, and 0.014 for alleles $\mathrm{TA}_{5}\left({ }^{*} 36\right), \mathrm{TA}_{6}\left({ }^{*} 1\right), \mathrm{TA}_{7}\left({ }^{*} 28\right)$, and $\mathrm{TA}_{8}\left({ }^{*} 37\right)$, respectively. Genotype frequencies were distributed according to those expected under HWE. The observed allele frequencies were not significantly different between ethnic groups. On the 375 patients evaluated, the majority of genotypes were $\mathrm{TA}_{6} / \mathrm{TA}_{6}, \mathrm{TA}_{6} / \mathrm{TA}_{7}$, and $\mathrm{TA}_{7} / \mathrm{TA}_{7}(n=353)$. Other genotypic combinations of $\mathrm{TA}_{5}$ and $\mathrm{TA}_{8}$ alleles were also present, but the rarer $\mathrm{TA}_{5} / \mathrm{TA}_{5}$ and $\mathrm{TA}_{8} / \mathrm{TA}_{8}$ genotypes were not observed. Due to the small number of individuals bearing $\mathrm{TA}_{5}$ and $\mathrm{TA}_{8}$ alleles, these rare allele combinations were excluded from the association analysis. A previous study of UGT1A1 (TA) $n$ polymorphism performed in the same geographic region but regarding hemolytic anemia patients and healthy controls ${ }^{12}$ found allelic and genotypic frequencies similar to those found herein.

The medians of total and indirect bilirubin levels in the whole sample and among the common genotypes are shown in Table 1. Bilirubin levels were not different among the three genotypes.

According to the AIDS Clinical Trials Group, severe hyperbilirubinemia was defined as total bilirubin levels $>3.1 \mathrm{mg} / \mathrm{dl}$, and $6.7 \%(n=25)$ of patients analyzed presented this outcome. From these 25 patients, $72 \%$ were atazanavir users and $60 \%$ carried at least one UGT1A1*28 allele.

The predicting variables that contributed to the development of severe hyperbilirubinemia were evaluated through multivariate Poisson regression analyses. The variables ethnic group, gender, and indinavir use were not significant contributors to this outcome, while the most parsimonious model included only atazanavir use, age, and presence of the UGT1A1*28 allele (Table 2).

Hepatic uridine diphosphate-glucuronosyltransferase catalyzes the conjugation of bilirubin with glucuronic acid to form the more water-soluble bilirubin diglucuronide, which is excreted into the bile. ${ }^{3}$ It is well established that the insertion of a TA dinucleotide in the TATA box of the UGT1A1 promoter results in an enzyme with reduced activity, leading to elevation of unconjugated bilirubin levels. However, correlation of bilirubin levels with the presence of the UGT1A1*28 allele is not necessarily obvious in all the populations studied ${ }^{3}$ due to several environmental factors that can also affect this

Table 2. Poisson Regression Models and Predicting Variables for Development of Severe Hyperbilirubinemia IN HIV-INFECTED INDIVIDUALS ON HAART

\begin{tabular}{|c|c|c|c|c|}
\hline Outcome & Predicting variable & $P R$ & $95 \% C I$ & $\mathrm{p}$ \\
\hline \multirow[t]{3}{*}{ Severe hyperbilirubinemia } & ATV use & 7.72 & $3.14-18.98$ & $<0.001$ \\
\hline & Age & 1.04 & $1.01-1.08$ & 0.029 \\
\hline & UGT1A1*28 allele & 2.43 & $1.08-5.45$ & 0.032 \\
\hline
\end{tabular}

PR, prevalence ratio; 95\% CI, confidence interval; ATV, atazanavir. 
phenotype, such as alcohol, drugs, smoking, age, and gender. Our results in a sample of HIV-infected individuals exposed to HAART are in line with these findings in general populations, as total and indirect bilirubin levels were only mildly elevated in $\mathrm{TA}_{7}$ allele homozygotes, being not significantly different among genotypes.

Moreover, the results of the multivariate Poisson regression analysis showed that the presence of the UGT1A1*28 allele is a significant risk factor for the development of the more extreme phenotype, severe hyperbilirubinemia, in HAART users. These results are in agreement with other studies ${ }^{5,6}$ and demonstrate that when we control for environmental variables that can also affect bilirubin levels, such as age and atazanavir use, the consequences of the presence of this risk allele can be highlighted. Furthermore, it is important to take into consideration that atazanavir is widely used as part of HAART, being associated with unconjugated hyperbilirubinemia due to the competition between this drug and the physiologic binding of bilirubin to the UGT1A1 enzyme. ${ }^{13}$

This is the first description of the influence of this gene variant on the development of severe hyperbilirubinemia in HIV-infected individuals in Brazil. This study is relevant especially because the Brazilian population is a very ethnically admixed population, which is the result of five centuries of interethnic crosses of peoples from three continents: the European colonizers, mainly represented by the Portuguese, the African slaves, and the autochthonous Amerindians. ${ }^{14}$ In the South of Brazil, where our study was performed, the Amerindian contribution is very low, and African influence is reduced in comparison to all other geographic regions. Published studies on this same gene evaluated a smaller number of patients and more ethnically restricted populations, such as Rodrigues-Novoa et al., which analyzed a total of 118 HIVinfected patients, all white; Rotger et al. evaluated 96 HIVinfected individuals, with $96 \%$ being of white ethnicity, and Anderson et al. analyzed $33 \mathrm{HIV}$-infected subjects, $79 \%$ of whom were white. ${ }^{5,6,15}$ Our data suggest that the effect of the UGT1A1*28 allele may also be observed in African and Euroamerican populations. This is even more important when we take into consideration the high number of HIV-infected individuals in these populations. In Brazil, it is estimated that about 630,000 people are infected with the virus. ${ }^{16}$

There are other UGT1A1 gene variants that have also been related to decreased enzymatic activity: UGT1A1*6 $(211 \mathrm{G}>\mathrm{A})$, UGT1A1*27 (686C > A), and UGT1A1*37 $\left(\mathrm{TA}_{8}\right)$. Although we have not analyzed the $* 6$ and $* 27$ alleles, according to previous studies, ${ }^{17}$ they have been found exclusively in Asian populations. Regarding the $* 37$ variant, in our sample we found only 11 carriers of this allele. Therefore, although this is a functional variant, we had no power to detect its effect due to the low number of individuals with this genotype.

The limitations of this study should also be considered, including its cross-sectional design and the inclusion of patients using different HAART regimens. However, due to the large number of drugs available for HIV therapy, it is very difficult to study a considerable number of patients on the same antiretroviral combinations. On the other hand, this heterogeneity allows us to highlight the effect of the UGT1A1*28 allele in the ATV-containing regimens in contrast to the other drug combinations.

In conclusion, the presence of the UGT1A1*28 allele with ATV use increases the risk of developing severe hyperbilir- ubinemia. Although hyperbilirubinemia is considered a mild adverse effect, it has clinical implications. Jaundice causes discomfort due to the yellowish appearance of the skin, which may affect the quality of life of these patients and may lead to treatment discontinuation. ${ }^{2,5}$ This finding is a good example of how pharmacogenomic studies can be useful and the consistency among findings in different populations indicates that perhaps the time has come to transfer these results from basic research to clinical practice. It is important to keep in mind that the variant allele frequencies should be considered in each population before initiating a genotyping program. Evidently, cost-effectiveness analyses are needed to determine the utility of genotyping as a screening measure previous to atazanavir use.

\section{Acknowledgments}

This study was supported by Departamento de DST, Aids e Hepatites Virais, Ministério da Saúde, Brazil, Conselho Brasileiro de Desenvolvimento Científico e Tecnológico, PRONEX/CNP $/$ FAPERGS and Coordenação de Aperfeiçoamento de Pessoal de Nível Superior.

\section{Author Disclosure Statement}

No competing financial interests exist.

\section{References}

1. Tozzi V: Pharmacogenetics of antiretrovirals. Antiviral Res 2010;85:190-200.

2. Hawkins T: Understanding and managing the adverse effects of antiretroviral therapy. Antiviral Res 2010;85:201-209.

3. Burchell B and Hume R: Molecular genetic basis of Gilbert's syndrome. J Gastroenterol Hepatol 1999;14:960-966.

4. Lankisch TO, Moebius U, Wehmeier M, et al.: Gilbert's disease and atazanavir: From phenotype to UDPglucuronosyltransferase haplotype. Hepatology 2006;44: 1324-1332.

5. Rotger M, Taffé P, Bleiber G, et al.: Gilbert syndrome and the development of antiretroviral therapy-associated hyperbilirubinemia. J Infect Dis 2005;192:1381-1386.

6. Rodriguez-Novoa S, Martín-Carbonero L, Barreiro P, et al.: Genetic factors influencing atazanavir plasma concentrations and the risk of severe hyperbilirubinemia. AIDS 2007; 21:41-46.

7. Baudhuin LM, Highsmith EW, Skierka J, Holtegaard L, Moore BE, and O'Kane DJ: Comparison of three methods for genotyping the UGT1A1 (TA) n repeat polymorphism. Clin Biochem 2007;40:710-717.

8. Fellay J, Boubaker K, Ledergerber B, et al.: Prevalence of adverse events associated with potent antiretroviral treatment: Swiss HIV Cohort Study. Lancet 2001;358:1322-1327.

9. Smiderle L, Galvão ACS, Fontana C, et al.: Evaluation of UGT1A1 and SULT1A1 polymorphisms with lipid levels in women with different hormonal status. Gynecol Endocrinol 2011;27:20-26.

10. Roff DA and Bentzen P: The statistical analysis of mitochondrial DNA polymorphisms: $\chi^{2}$ and the problem of small samples. Mol Biol Evol 1989;6:539-545.

11. Zembrzuski VM, Callegari-Jacques SM, and Hutz MH: Application of an African Ancestry Index as a genomic control approach in a Brazilian population. Ann Hum Genet 2006;70:822-828.

12. Azevedo LA, Santin AP, Wagner SC, Zaleski CF, Bock H, Saraiva-Pereira ML, and Castro SM: Prevalence of UGT1A1 
gene polymorphism in patients with hemolytic anemia in southern Brazil. Genet Test Mol Biomarkers 2011;15:107-110.

13. Rodriguez-Novoa S, Barreiro P, Jimenez-Nacher I, and Soriano V: Overview of the pharmacogenetics of HIV therapy. Pharmacogenom J 2006;6:234-245.

14. Parra FC, Amado RC, Lambertucci JR, Rocha J, Antunes $\mathrm{CM}$, and Pena SDJ: Color and genomic ancestry in Brazilians. Proc Natl Acad Sci USA 2003;100:177-182.

15. Anderson PL, Lamba J, Aquilante CL, Schuetz E, and Fletcher $\mathrm{CV}$ : Pharmacogenetic characteristics of indinavir, zidovudine, and lamivudine therapy in HIV-infected adults: a pilot study. J Acquir Immune Defic Syndr 2006;42:441-449.

16. Brasil. Ministério da Saúde. Secretaria de Vigilância em Saúde. Programa Nacional de DST e Aids. Boletim Epidemiológico 2010. Available at http://www.aids.gov.br/ publicacao/boletim-epidemiologico-2010. Accessed 06/ 30/2011.

17. Pharmacogenomics Knowledge Base (PharmGKB). Available at http://www.pharmgkb.org/images/header/title.png. Accessed 10/07/2011.

Address correspondence to: Vanessa S. Mattevi

Rua Sarmento Leite 245 sala 309 CEP 90050-170 Porto Alegre, RS Brazil

E-mail: vmattevi@ufcspa.edu.br 